\title{
Lipid remodeling under acidic conditions and its interplay with low Pi stress in Arabidopsis
}

\author{
Masato Murakawa $^{1} \cdot$ Hiroyuki Ohta $^{1} \cdot$ Mie Shimojima $^{1}$ (D)
}

Received: 12 March 2019 / Accepted: 6 June 2019 / Published online: 14 June 2019

(c) The Author(s) 2019

\begin{abstract}
Key message Here we show that accumulation of galactose-containing lipids in plastid membranes in shoots and the other membranes in roots maintains Arabidopsis growth under acidic stress and acidic phosphate deficiency.

Abstract Soil acidification and phosphate deficiency are closely related to each other in natural environments. In addition to the toxicity of high proton concentrations, acid soil can lead to imbalances of ion availability and nutritional deficiencies, including inorganic phosphate (Pi). Among plants, activation of non-phosphorus-containing galactolipid, digalactosyldiacylglycerol (DGDG), synthesis concomitant with phospholipid degradation, namely membrane lipid remodeling, is crucial for coping with Pi starvation. However, regulation mechanisms of membrane lipid composition during acidic stress have not been clarified. Here, we investigated lipid metabolism in Arabidopsis thaliana grown under acidic stress with or without Pi. Under Pi-sufficient acidic conditions, DGDG was increased in shoot membranes, and some Pi starvation-responsive genes that are involved in lipid remodeling were upregulated without reducing Pi content in leaves. In contrast, under acidic Pi deficiency, membrane lipid remodeling in roots was partially repressed at a lower external $\mathrm{pH}$. Nevertheless, phenotypic comparison between wild type and the double mutant of MGD2/3, which are responsible for DGDG accumulation during Pi starvation, indicated that the complete absence of lipid remodeling in roots resulted in a loss of tolerance to Pi deficiency rather specifically under acidic conditions. This result suggested important physiological roles of galactolipid-enriched membranes under acidic Pi deficiency.
\end{abstract}

Keywords Galactolipid · Digalactosyldiacylglycerol (DGDG) · MGDG synthase (MGD) · Lipid remodeling · Phosphate starvation $\cdot$ Acidic stress

\section{Introduction}

Soil acidification is a major agricultural problem that affects plant growth on a worldwide scale (von Uexküll and Mutert 1995; Shavrukov and Hirai 2016). In acid soils, which are defined as having a pH below 5.0-5.5, an increase in the concentration of protons can limit the growth of plants and also causes other associated stresses; an elution of toxic ions such as aluminum (Al) and manganese from the soil, a

Electronic supplementary material The online version of this article (https://doi.org/10.1007/s11103-019-00891-1) contains supplementary material, which is available to authorized users.

Mie Shimojima

shimojima.m.aa@m.titech.ac.jp

1 School of Life Science and Technology, Tokyo Institute of Technology, 4259-B-65 Nagatsuta-cho, Midori-ku, Yokohama, Kanagawa 226-8501, Japan decrease in available inorganic phosphate ( $\mathrm{Pi})$, molybdenum, calcium, potassium, and magnesium. Pi bioavailability in the soil is often limited by forming insoluble salts with iron or $\mathrm{Al}$, especially in acid soils. Inversely, rhizosphere acidification caused by plants has been reported to occur under Pi deficiency, most likely because of exudation of protons and organic acids and/or imbalances in the uptake of ions (Hinsinger et al. 2003). Thus, for plants in nature, acidic environment is highly associated with Pi deficiency.

Low-pH conditions can irreversibly inhibit the root growth of plants both quickly and severely because of the toxicity of the protons themselves (Koyama et al. 2001). As regards the acidic stress tolerance of plants, during the early phase of exposure to low-pH environments, membranelocalized proton transporters $\left(\mathrm{H}^{+}\right.$-ATPases) are activated and pump out excess protons from the cytoplasm to the extracellular space or sequester them in vacuoles (Yan et al. 1998). With longer-term exposure, detoxification systems for 
reactive oxygen species and signaling in response to biotic and abiotic stresses, including defense-associated hormone signaling, are induced (Lager et al. 2010; Hachiya et al. 2014). In addition, auxin signaling also contributes to the adaptation to acidic stress through activating a $\mathrm{H}^{+}$-ATPase localized in the plasma membrane (Inoue et al. 2016). Although membranes are thought to be impaired by the lipid peroxidation during acidic stress (Zhang et al. 2015), the membrane lipid composition of plants grown under acidic conditions has not yet been analyzed in detail.

Upon Pi starvation, plants alter the membrane lipid composition, called membrane lipid remodeling. To provide Pi for essential biological processes, a substantial portion of phospholipids is degraded and replaced by non-phosphorus lipids (Härtel et al. 2000; Nakamura 2013). In seed plants, Pi starvation enhances accumulation of digalactosyldiacylglycerol (DGDG), which is synthesized on the plastidial envelope membranes and is transported to the extraplastidial membranes: the plasma membrane, mitochondrial membrane, and tonoplast membranes (Andersson et al. 2003, 2005; Jouhet et al. 2004). Under Pi-sufficient conditions, however, galactolipids mainly exist in the plastids. Two kinds of galactolipids, DGDG and monogalactosyldiacylglycerol (MGDG), constitute 26 and 52\% of thylakoid membrane from spinach chloroplasts, respectively (Block et al. 1983). These galactolipids are synthesized by MGDG synthases (MGDs) and DGDG synthases (DGDs). MGD transfers a galactosyl moiety from UDP-galactose to diacylglycerol to form MGDG. Subsequently, DGD synthesizes DGDG by galactosylation of MGDG using UDP-galactose as the substrate. Arabidopsis has three MGDs and two DGDs, MGD1/2/3 and DGD1/2, respectively (Benning and Ohta 2005). The three MGDs are generally classified as type-A (MGD1) and type-B (MGD2/3) (Miège et al. 1999; Awai et al. 2001). The type-A MGD is localized on the inner envelope membrane of plastids and functions together with DGD1; the MGD1-DGD1 pathway is carried out mainly in green tissues and is responsible for the accumulation of the bulk of galactolipids in chloroplast membranes. In the shoots of Arabidopsis MGD1 knock-out mutant, the amount of MGDG was reduced by $~ 98 \%$ compared with the wild-type (WT), and DGDG was also scarcely detected (Kobayashi et al. 2007). On the other hand, double knockout mutant of MGD2/3, $m g d 2 m g d 3$, showed comparable amount of galactolipids to WT in shoots under Pi-sufficient conditions (Kobayashi et al. 2009a, b). Type-B MGDs are localized on the outer envelope membrane of plastids and function together with DGD2; the MGD2/3-DGD2 pathway contributes to galactolipid synthesis in non-photosynthetic tissues such as roots and flowers (Awai et al. 2001; Nakamura et al. 2009a). Furthermore, the MGD2/3-DGD2 pathway is highly induced under Pi deficiency and is involved in membrane lipid remodeling under such conditions. Lipid analyses on $m g d 2 m g d 3$ indicated that type-B MGDs contribute $~ 50 \%$ of DGDG increase respond to Pi-starvation in shoots. In contrast, DGDG accumulation in roots respond to Pi-starvation is almost entirely dependent on MGD2/3-DGD2 pathway (Kobayashi et al. 2009a, b). Although the metabolic pathways of galactolipids in Arabidopsis have been investigated in detail (Shimojima and Ohta 2011), the regulation of galactolipid synthesis under acidic conditions remains unknown. In this study, we report that membrane lipid composition is altered under acidic conditions with or without $\mathrm{Pi}$, and membrane lipid remodeling is possibly involved in the stress adaptation to acidic Pi deficiency.

\section{Materials and methods}

\section{Plant materials and growth conditions}

WT Arabidopsis thaliana was of the Columbia ecotype (Col-0). The double-knockout mutant of type-B MGDG synthases, $m g d 2 m g d 3$, was obtained as described (Kobayashi et al. 2009a, b). Sterilized seeds were plated on halfstrength Murashige and Skoog (MS) medium (Murashige and Skoog 1962) containing 1\% (w/v) sucrose, $20 \mathrm{mM}$ MES-KOH at $\mathrm{pH} 6.0$ and $0.8 \%(\mathrm{w} / \mathrm{v})$ agar. Seeds were incubated at $4{ }^{\circ} \mathrm{C}$ for 4 days in the dark and germinated under continuous white light $\left(\sim 50 \mu \mathrm{E} \mathrm{m}^{-2} \mathrm{~s}^{-1}\right)$ at $23{ }^{\circ} \mathrm{C}$ for 10 days. Then, plants were transferred to three-fold diluted MGRL medium (1/3 MGRL) at $\mathrm{pH} \sim 6.0$ (Fujiwara et al. 1992) for 5 days. For the stress treatments, plants were subsequently transferred to $1 / 3$ MGRL medium, which was adjusted to $\mathrm{pH} 3.7$ by using $\mathrm{H}_{2} \mathrm{SO}_{4}$ or $\mathrm{HCl}$, and grown for another 7-9 days. The $\mathrm{pH}$ of the acidic medium was monitored and adjusted every day. For the control conditions, plants were transferred to $1 / 3$ MGRL medium without adjusting the $\mathrm{pH}$ (initially at $\mathrm{pH} \sim 6.0$ ). For Pi depletion, 1/3 MGRL medium without phosphate containing $10 \mathrm{mM}$ MES-KOH at $\mathrm{pH} 6.0$ was used for plant hydroponic culture as described above, whereas the $\mathrm{pH}$ was adjusted to 4.5 or 3.7 by using $\mathrm{H}_{2} \mathrm{SO}_{4}$ for the acidic Pi-deficient conditions.

\section{Estimation of ion concentrations in plant growth media}

Ion concentrations in 1/3 MGRL medium with or without phosphate at $\mathrm{pH} 6.0$ and 3.5 were calculated using GEOCHEM-EZ (Shaff et al. 2010). Parameters were set as follows: "Convergence criterion" was set at 1e-4; "Number of Interactions" was set at 50; "Solids" was set at "can precipitate"; "Fixed Ionic Strength" was set at 0.10. 


\section{Lipid analysis}

Lipid analyses were done essentially as described (Kobayashi et al. 2007). Total lipids were extracted from 22-daysold plant samples as described (Bligh and Dyer 1959). Polar lipids were separated by two-dimensional TLC on silica gel TLC plates (TLC Silica gel 60 , Glass plates $20 \times 20 \mathrm{~cm}$; Merck) using the following solvent systems: chloroform/ methanol/7 M ammonia (115:80:8, v/v/v) for the first dimension and chloroform/methanol/acetic acid/water $(170: 25: 15: 3, \mathrm{v} / \mathrm{v} / \mathrm{v} / \mathrm{v})$ for the second dimension. Separated lipids were derivatized to fatty acid methyl esters and quantified by gas chromatography using pentadecanoic acid as an internal standard. Preparation of oligogalactolipids was performed as described (Barnes et al. 2016). Polar lipids were separated by TLC using the following solvent system: chloroform/methanol/acetic acid/water (85:20:10:4, v/v/v/v). Carbohydrates in the head group of lipids were visualized by spraying with a solvent $(2.5 \mathrm{mM}$ anthrone, $1.3 \mathrm{mM}$ thiourea, $66 \%(\mathrm{v} / \mathrm{v})$ sulfuric acid) and baking at $110{ }^{\circ} \mathrm{C}$ for $10 \mathrm{~min}$. For the positive control of this analysis, an SFR2 assay in whole-plant tissue was done as described (Barnes et al. 2016). Briefly, a 14-days-old WT Arabidopsis rosette was floated on the assay buffer ( $20 \mathrm{mM}$ acetic acid, $10 \mathrm{mM}$ $\mathrm{MgCl}_{2}$, adjusted to $\mathrm{pH} 4.0$ with $\mathrm{K}_{2} \mathrm{HPO}_{4}$ ) overnight under dim light.

\section{qPCR}

Total RNA was extracted from 22-days-old plant samples using the SV Total RNA Isolation System (Promega). Reverse transcription was performed with $325 \mathrm{ng}$ RNA using the PrimeScript RT reagent kit (Takara). qPCR was carried out using the SYBR Premix Ex Taq II (Takara) based on the manufacturer's protocols, and signals were detected with the Thermal Cycler Dice Real Time System (Takara). The $2^{-\Delta \Delta C t}$ value was calculated based on the expression of $U B Q 10$ as an internal standard. Sequences of primers are listed (Table SIII) as described (Bari et al. 2006; Murakawa et al. 2014).

\section{Isolation of microsomal membrane fraction}

The microsomal membrane fraction was obtained essentially as described (Nakamura et al. 2009b; Murakawa et al. 2014). Shoots from 22-day-old plants were homogenized in the buffer (50 mM HEPES-KOH at pH 7.8, 2 mM EDTA, $1 \mathrm{mM} \mathrm{MgCl}_{2}, 1 \mathrm{mM} \mathrm{MnCl}_{2}$ ), and tissue debris was removed by centrifugation at $300 \times g$. To remove thylakoid membranes, the crude extract was centrifuged twice at $3000 \times g$ for $10 \mathrm{~min}$, and the resulting supernatant was centrifuged at $125,000 \times g$ for $60 \mathrm{~min}$ to separate the microsomal fraction as a pellet from the soluble fraction. The microsomal fraction was suspended in $50 \mathrm{mM}$ HEPES-KOH at $\mathrm{pH} 7.8$, and polar lipids were extracted from the microsomal fraction by addition of $10 \mathrm{vol}$ of chloroform/methanol $(2: 1, \mathrm{v} / \mathrm{v})$.

\section{Measurement of Pi content}

Pi was extracted from 22-days-old plant samples and quantified by phosphomolybdate colorimetric assay as described (Chiou et al. 2006). Briefly, samples were homogenized with extraction buffer $(10 \mathrm{mM}$ Tris, $1.0 \mathrm{mM}$ EDTA, $100 \mathrm{mM}$ $\mathrm{NaCl}, 1.0 \mathrm{mM} \beta$-mercaptoethanol, $\mathrm{pH}$ 8.0) and centrifuged at $12,000 \times \mathrm{g}$ for $10 \mathrm{~min}$. A portion of the resulting supernatant $(100 \mu \mathrm{L})$ was mixed with $900 \mu \mathrm{L}$ of $1 \%(\mathrm{v} / \mathrm{v})$ glacial acetic acid and incubated at $42{ }^{\circ} \mathrm{C}$ for $30 \mathrm{~min}$. The mixture was then centrifuged at $12,000 \times g$ for $5 \mathrm{~min}$. A portion of the resulting supernatant $(300 \mu \mathrm{L})$ was mixed with $700 \mu \mathrm{L}$ of assay solution $\left(0.35 \%\right.$ [w/v] $\mathrm{NH}_{4} \mathrm{MoO}_{4}, 0.43 \mathrm{M} \mathrm{H}_{2} \mathrm{SO}_{4}$, $1.4 \%$ [w/v] ascorbic acid) and incubated at $42{ }^{\circ} \mathrm{C}$ for $30 \mathrm{~min}$. The Pi content was measured at $A_{820}$ based on the calibration curve obtained from the Pi standard solutions.

\section{Liposome preparation}

Total lipids were extracted from spinach as described (Bligh and Dyer 1959) and separated by TLC using a solvent system consisting of acetone/toluene/water (136:45:12, v/v/v). Spinach DGDG and PC were eluted from the silica gel by using chloroform/methanol $(1: 1, \mathrm{v} / \mathrm{v})$ and methanol, respectively. To form liposomes, $2 \mathrm{mg}$ of DGDG and/or PC was mixed with $2 \mathrm{mg}$ of lecithin, which was prepared by removing proteins and triacylglycerol from Asolectin from soybean (Sigma) with chloroform/iced acetone (1:6, v/v). Each mixture of lipid suspension was dried under nitrogen gas in a glass test tube and re-hydrated with $300 \mu \mathrm{L}$ of buffer solution ( $2 \mathrm{mM}$ pyranine, $5 \mathrm{mM} \mathrm{KH}{ }_{2} \mathrm{PO}_{4}, 100 \mathrm{mM} \mathrm{KCl}, 1 \mathrm{nmol}$ valinomycin/mg.lipid, adjusted to $\mathrm{pH} 7.0$ with $\mathrm{KOH}$ ) at $37^{\circ} \mathrm{C}$ for $10 \mathrm{~min}$. Then, a liposome suspension was prepared by sonication using an ultrasonic cleaner (Branson 3210, Yamato) for $20 \mathrm{~min}$ and an ultrasonic disruptor (UD-201, TOMY: output, 1; duty, 30) for $60 \mathrm{~s}$. To remove disordered liposomes, each suspension was centrifuged at $3000 \times g$ for $5 \mathrm{~min}$. Then, each liposome suspension was dialyzed against pyranine-free buffer $\left(5 \mathrm{mM} \mathrm{KH}_{2} \mathrm{PO}_{4}, 100 \mathrm{mM} \mathrm{KCl}\right.$, adjusted to $\mathrm{pH} 7.0$ with $\mathrm{KOH}$ ) to remove pyranine present outside of the liposomes.

\section{Measurement of proton permeability of liposomes}

The measurement of proton permeability of the liposomes was conducted as described (Shimada et al. 2008), with some modifications. The internal $\mathrm{pH}$ of the liposomes was estimated based on the fluorescence excitation spectrum of pyranine (Kano and Fendler 1978). Each liposome 
suspension $(100 \mu \mathrm{L})$ was mixed with $1.9 \mathrm{~mL}$ of acidic buffer (5 mM KH${ }_{2} \mathrm{PO}_{4}, 100 \mathrm{mM} \mathrm{KCl}$, adjusted to $\mathrm{pH} 4.5$ with $\mathrm{HCl}$ ), and the excitation spectrum of fluorescence emission at $510 \mathrm{~nm}$ was measured for $30 \mathrm{~min}$ with a fluorescence spectrophotometer (F-2700, HITACHI). For the intensity at $0 \mathrm{~min}, 100 \mu \mathrm{L}$ of liposome suspension was mixed with $1.9 \mathrm{~mL}$ of neutral buffer $\left(5 \mathrm{mM} \mathrm{KH}{ }_{2} \mathrm{PO}_{4}, 100 \mathrm{mM} \mathrm{KCl}\right.$, adjusted to $\mathrm{pH} 7.0$ with $\mathrm{KOH}$ ). The internal $\mathrm{pH}$ of the liposomes was calculated based on the logarithm of the ratio of emission intensities with excitation at 400 and $460 \mathrm{~nm}$ : $\log \left(I_{400 \mathrm{~nm}} / I_{460 \mathrm{~nm}}\right)$. To obtain the calibration curve in Figure S1, $\log \left(I_{400 \mathrm{~nm}} / I_{460 \mathrm{~nm}}\right)$ of standard solutions $(33 \mu \mathrm{M}$ pyranine; $5 \mathrm{mM} \mathrm{KH}_{2} \mathrm{PO}_{4} ; 100 \mathrm{mM} \mathrm{KCl}$; at $\mathrm{pH} 4.5,5.0,5.5,6.0$, $6.5,7.0$ and 7.5) was plotted against the solution $\mathrm{pH}$.

\section{Measurement of chlorophyll content}

Total chlorophyll was extracted in $80 \%(\mathrm{v} / \mathrm{v})$ acetone at $4{ }^{\circ} \mathrm{C}$. Samples were centrifuged at $12,000 \times g$ for $5 \mathrm{~min}$, and the supernatant was used for the measurement of the absorbance with a spectrophotometer (UV-1850, Shimadzu). Total chlorophyll was calculated using the following formula (Porra 2002): Total chlorophyll $\left(\mathrm{nmol} \cdot \mathrm{mL}^{-1}\right)=19.54 \times\left(A_{646.8}-\right.$ $\left.A_{720}\right)+8.29 \times\left(A_{663.2}-A_{720}\right)$.

\section{Results}

\section{Lipid metabolism in WT Arabidopsis grown under acidic conditions}

To assess the effects of acidic conditions on plant growth, we investigated morphological phenotypes of WT Arabidopsis grown at $\mathrm{pH}$ 3.7. To compare the effects of the counteranion of the acid, we adjusted the medium $\mathrm{pH}$ with $\mathrm{H}_{2} \mathrm{SO}_{4}$ or $\mathrm{HCl}$. $\mathrm{SO}_{4}{ }^{2-}$ is not effective at permeating membranes, whereas $\mathrm{Cl}^{-}$is highly permeating (Hinsinger et al. 2003). As compared with plants grown under control conditions, rosette leaf expansion and primary root elongation were arrested at pH 3.7 (Fig. 1). As for the comparison between $\mathrm{H}_{2} \mathrm{SO}_{4}$ and $\mathrm{HCl}$, there were no obvious differences in either the shoot or root phenotype, showing that the protons released from the acids, but not the counteranions, were responsible for growth impairment under acidic conditions. To clarify whether the increase in the proton concentration would cause changes in ion balances in the growth medium, we calculated the ion concentrations in our growth medium at $\mathrm{pH} 6.0$ and 3.5 with the chemical speciation program GEOCHEM-EZ (Shaff et al. 2010). Based on this simulation, insoluble salts that prevent plants absorbing nutrients are not formed either at pH 6.0 or pH 3.5 (Table SI), indicating that plants do not (a)

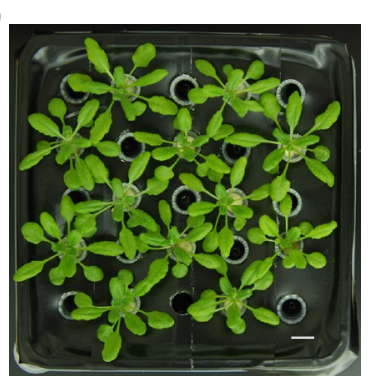

(b)

(c)
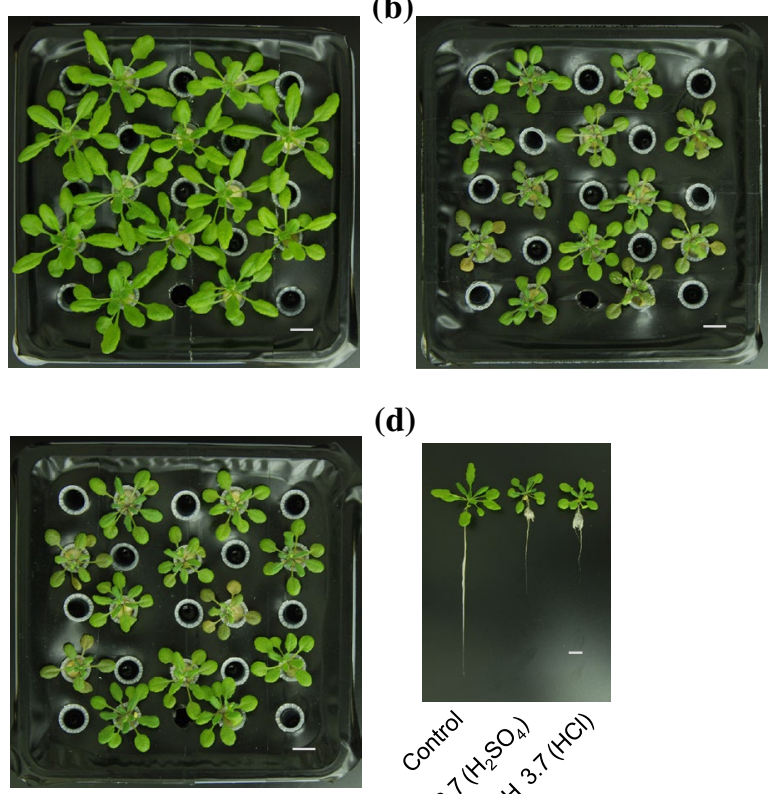

(d)

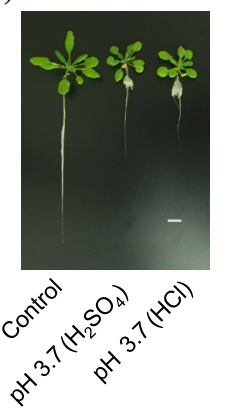

(e)

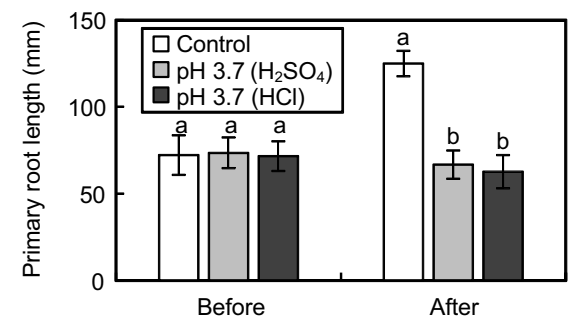

Fig. 1 Growth phenotypes of WT Arabidopsis plants under acidic conditions. Plant seedlings were germinated on $1 / 2$ MS medium for 10 days and then transferred to 1/3 MGRL medium for 5 days. Acidic stress treatments were subsequently conducted; Plants were grown in 1/3 MGRL medium as the control (a) or acidic 1/3 MGRL medium at $\mathrm{pH} 3.7$ adjusted with $\mathrm{H}_{2} \mathrm{SO}_{4}$ (b) or $\mathrm{HCl}$ (c) for another 7 days. d Whole morphology of WT Arabidopsis grown under acidic conditions. Bars $=1 \mathrm{~cm}$. e Primary root length of WT Arabidopsis before and after a week of acidic stress treatment. Values are the mean \pm SD $(n=20)$. Different letters represent significant differences based on the Tukey-Kramer multiple comparison test $(p<0.05)$

suffer from nutritional deprivation under acidic conditions in our experimental protocol.

To examine whether membrane lipid composition is altered in response to acidic stress, lipid analyses were conducted with shoots and roots of WT Arabidopsis grown at $\mathrm{pH}$ 3.7, which was adjusted with $\mathrm{H}_{2} \mathrm{SO}_{4}$ or $\mathrm{HCl}$ (Fig. 2). Similar to the morphological phenotypes, there were no significant differences in lipid composition between the two kinds of acidic stresses, $\mathrm{H}_{2} \mathrm{SO}_{4}$ and $\mathrm{HCl}$. In shoots of WT plants grown under acidic conditions, the molar percentage of DGDG was significantly increased by 25\% as compared with plants grown under control conditions 
Fig. 2 Lipid, gene expression and Pi content analyses of WT Arabidopsis plants grown under acidic conditions. Seedlings were germinated and grown as in Fig. 1. Composition of polar membrane lipids in shoots (a) and roots (b). SQDG, sulfoquinovosyldiacylglycerol; PI, phosphatidylinositol; PA, phosphatidic acid. Values are the mean $\pm \mathrm{SD}(n=3)$. c Gene expression analyses in rosette leaves. Relative mRNA abundance of each gene in expanded rosette leaves was analyzed by qPCR using UBQ10 as an internal control. Values are the mean $\pm \mathrm{SD}(n=8)$ of expression fold differences relative to that of control conditions. d Pi content in rosette leaves. Values are the mean \pm SD $(n=4)$. Different letters represent significant differences based on the Tukey-Kramer multiple comparison test $(p<0.05)$

(Fig. 2a). Meanwhile, phosphatidylcholine (PC) and phosphatidylethanolamine (PE), which are considerably degraded under Pi starvation, were not decreased under acidic conditions. Instead, MGDG and phosphatidylglycerol (PG) were slightly decreased. In roots, no marked change in molar percentages of galactolipids in response to acidic stress was observed (Fig. 2b). Fatty acid composition of lipids in shoots and roots of WT under acidic conditions is shown in Figs. S1 and S2, respectively. There were no marked differences in fatty acid composition of MGDG and DGDG under acidic conditions, suggesting that the contribution of MGD1, MGD2 and MGD3 to MGDG and DGDG synthesis under acidic conditions seems to be comparable between control and acidic conditions.

We also examined transcriptional changes in leaves from WT plants grown under acidic conditions by qPCR (Fig. 2c). With respect to MGDG synthase genes, MGD1 expression was significantly downregulated, whereas $M G D 2$ and $M G D 3$ were significantly upregulated. As for DGDG synthase genes, expression of both $D G D 1$ and $D G D 2$ was increased, but $D G D 1$ was more notably increased by $\sim$ twofold. Because $M G D 2 / 3$ and $D G D 1 / 2$ are inducible under Pi starvation (Awai et al. 2001; Kelly and Dörmann 2002; Kelly et al. 2003), we also analyzed the expression of $A t 4$, which is one of the Pi starvation marker genes but is not involved in lipid remodeling (Martín et al. 2000). At4 transcripts were significantly increased under acidic conditions, suggesting that a part of the Pi starvation response is induced at the transcriptional level by acidic stress. In addition, the expression of an auxin-responsive gene, IAAl, was also upregulated, consistent with the previous evidence that acidic stress induces auxin accumulation and auxin response signaling (Lager et al. 2010; Hachiya et al. 2014). NPC4/5 and PAH1/2 are involved in phospholipid degradation and supply substrates for galactolipid synthesis under Pi-deficient conditions (Nakamura et al. 2005; Gaude et al. 2008; Nakamura et al. 2009b). Although NPC4/5 and PAHI are Pi starvation-responsive genes, only $P A H 1$ expression was increased under acidic conditions, suggesting that acidic stress does not activate all aspects of Pi starvation-inducible phospholipid degradation. These results are consistent with the lipid analysis
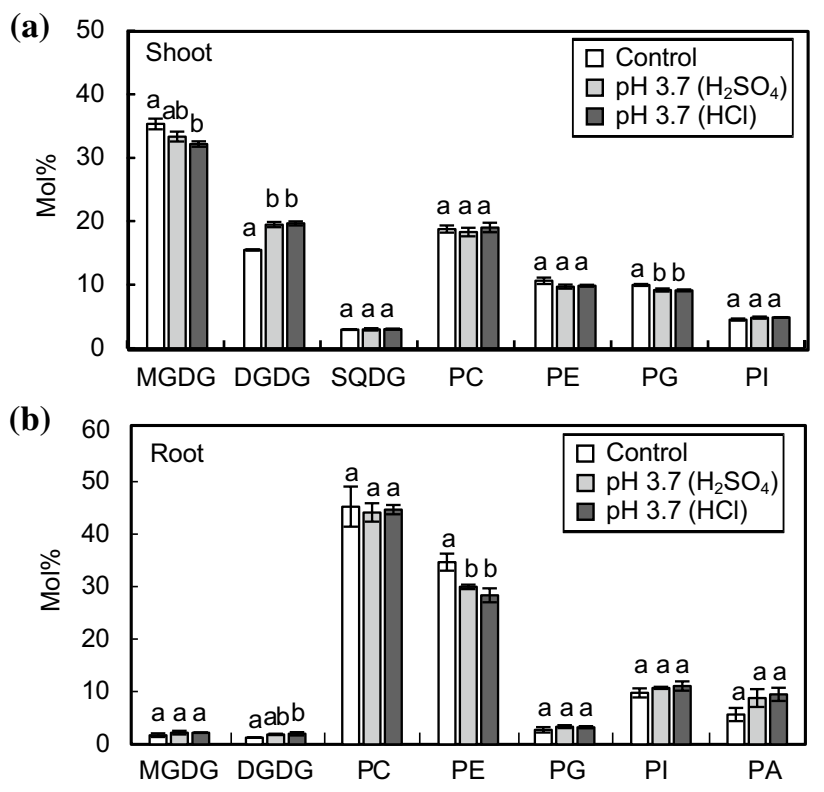

(c)
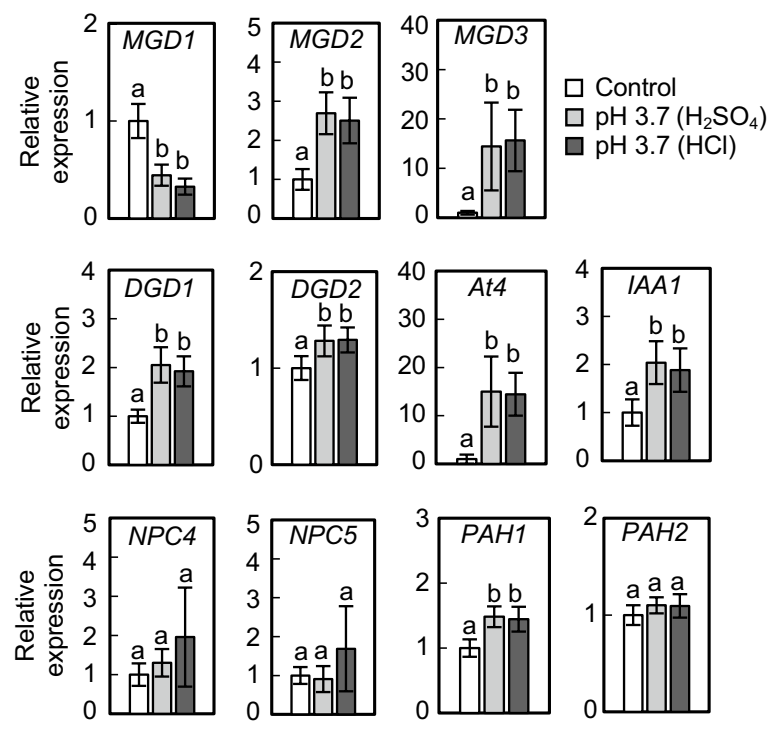

(d)

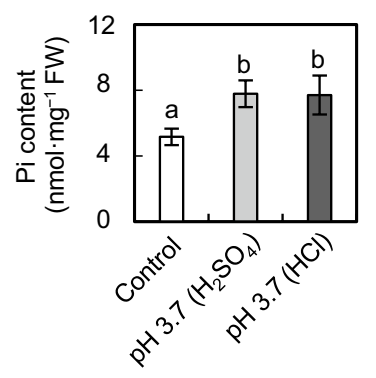

(Fig. 2a). There were no significant differences in gene expression patterns between medium containing $\mathrm{H}_{2} \mathrm{SO}_{4}$ and $\mathrm{HCl}$, in agreement with our results for plant growth phenotypes and lipid composition. 
To confirm whether or not plants had sufficient Pi under acidic conditions, the $\mathrm{Pi}$ content in leaves was measured (Fig. 2d). The results showed that Pi concentration was not decreased, rather increased, at $\mathrm{pH} 3.7$, indicating that plants were not Pi starved during acidic stress with either $\mathrm{H}_{2} \mathrm{SO}_{4}$ or $\mathrm{HCl}$.

\section{Lipid metabolism in mgd2mgd3 Arabidopsis grown under acidic conditions}

For further investigation of lipid metabolism during acidic stress, we grew $m g d 2 m g d 3$, the double knockout mutant of type-B MGD genes, under acidic conditions. Defects in $M G D 2 / 3$ did not lead to obvious changes in growth phenotypes under acidic conditions, compared to WT (Fig. 3a, b). As for lipid compositions, $m g d 2 m g d 3$ also showed the same pattern as WT (Figs. 2a, b, 3c, d). These results clearly indicated that, unlike Pi-depleted conditions, MGD2 and MGD3 were not involved in the DGDG accumulation but MGD1DGD1 pathway was responsible for DGDG accumulation in shoots under acidic conditions. To further examine the accumulation site of DGDG in shoots under acidic conditions, microsomal membranes were obtained from shoots of WT and $m g d 2 m g d 3$ grown at pH 3.7 adjusted with $\mathrm{H}_{2} \mathrm{SO}_{4}$, and their galactolipid content was analyzed (Fig. 3e). The microsomal membranes obtained by differential centrifugation mainly consist of the plasma membrane and membranes derived from the endoplasmic reticulum, Golgi apparatus, trans-Golgi network, tonoplast, mitochondria and the envelope membranes of chloroplasts. Thus, the overall amount of MGDG and DGDG, which are the main constituents of thylakoid membrane, was decreased in the microsomal membranes as compared with the total membrane lipids (Figs. 2a, 3c, e). However, the amounts of DGDG in microsomal membranes of both WT and $m g d 2 m g d 3$ grown under acidic conditions were significantly increased by $\sim 35 \%$ as compared with that of control conditions, whereas MGDG showed no increase. These results suggest that, under acidic stress, DGDGs were accumulated in the chloroplast envelope membranes or the extraplastidic membranes similarly to Pi starvation.

In addition to the two MGD-DGD pathways (MGD1DGD1 pathway and MGD2/3-DGD2 pathway) plants have an alternative galactolipid synthetic mechanism, galactolipid:galactolipid galactosyltransferase (GGGT), using MGDG as the donor of a galactose moiety (Dörmann and Benning 2002). In Arabidopsis, it has been suggested that cytosolic acidification and $\mathrm{Mg}^{2+}$ leakage from chloroplasts caused by freezing stress triggers GGGT activation and synthesis of oligogalactolipids, mainly trigalactosyldiacylglycerol (TGDG) and tetragalactosyldiacylglycerol (TeGDG) (Moellering et al. 2010; Barnes et al. 2016). Thus, we analyzed oligogalactolipids from WT and $m g d 2 m g d 3$ grown under acidic conditions (Fig. 3f). MGDG, DGDG and sulfoquinovosyldiacylglycerol (SQDG) were detected, yet neither TGDG nor TeGDG were visible, indicating that oligogalactolipids that contain three or more sugar units did not accumulate in Arabidopsis membranes under acidic conditions, indicating that TGDG synthesis catalyzed by GGGT is not activated under acidic conditions.

We also examined transcriptional changes in leaves from $m g d 2 m g d 3$ plants grown under acidic conditions by qPCR (Fig. 3g). Expression of $M G D 1, D G D 1$ and DGD2 in $m g d$ $2 m g d 3$ was similar to those in WT both under control and acidic conditions, suggesting that an increase in DGDG under acidic conditions in $m g d 2 m g d 3$ is not due to the upregulation of these gene expression compared with WT.

\section{Lipid metabolism in WT Arabidopsis grown under acidic Pi deficiency}

As acidic stress induced the expression of some Pi starvation-responsive genes (Fig. 2c), we further examined how a combined stress of an acidic environment under Pi deficiency affects the physiology of Arabidopsis. We confirmed that a change in $\mathrm{pH}$ from 6.0 to 3.5 does not cause the formation of insoluble salts in Pi-depleted growth medium (Table SII). Then, we observed the morphological phenotypes of WT Arabidopsis grown under Pi-deficient conditions at pH $6.0,4.5$ or 3.7 (Fig. 4). The size of rosette leaves was comparable among the three $\mathrm{pH}$ conditions under $\mathrm{Pi}$ deficiency, whereas the primary root growth was repressed with a decrease in $\mathrm{pH}$ (Fig. 4d, e), suggesting that plants have the ability to modify their root growth depending on external $\mathrm{pH}$ conditions even under Pi deficiency.

We subsequently conducted lipid analyses of WT Arabidopsis grown under acidic Pi-deficient conditions (Fig. 5a, b). We confirmed Pi starvation-induced lipid remodeling in both shoots and roots when compared the Pi-starved seedlings for 5 days (before) with those for 12 days (-Pi, $\mathrm{pH}$ 6.0) as shown in Fig. 5a and b. In shoots, the effect of acidic stress under Pi deficiency was not remarkable and the lipid compositions of seedlings under three different $\mathrm{pH}$ conditions (-Pi, $\mathrm{pH} 6.0, \mathrm{pH} 4.5$ and $\mathrm{pH}$ 3.7) were comparable (Fig. 5a). In roots, by contrast, the amount of DGDG at a lower $\mathrm{pH}(\mathrm{pH} 4.5$ and $\mathrm{pH} 3.7$ ) was significantly lower than that at pH 6.0 (Fig. 5b), indicating that the Pi starvationinduced lipid remodeling in roots was partially repressed by acidic stress. Fatty acid composition of galactolipids in shoots and roots under acidic Pi deficiency showed no obvious change (Figs. S3, S4).

We also performed qPCR analyses in rosette leaves and roots from WT Arabidopsis grown under acidic Pideficient conditions (Fig. 5c, d). It should be noted that the base expression level, at $\mathrm{pH}$ 6.0, of $\mathrm{Pi}$ starvation-responsive genes, especially $M G D 2 / 3, D G D 1 / 2, A t 4, N P C 4 / 5$ and 
(a)

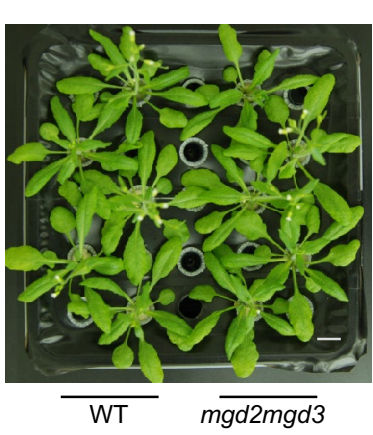

(b)

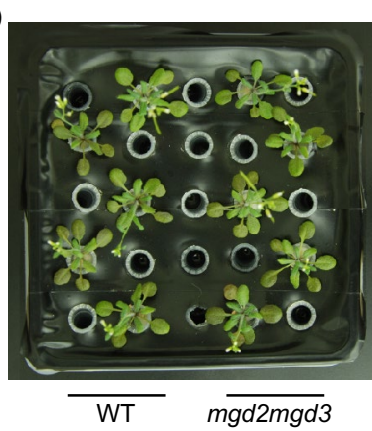

(f)

(g)

(c)

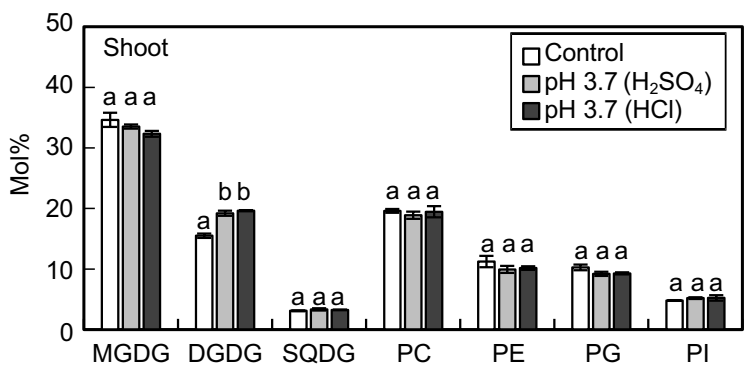

(d)

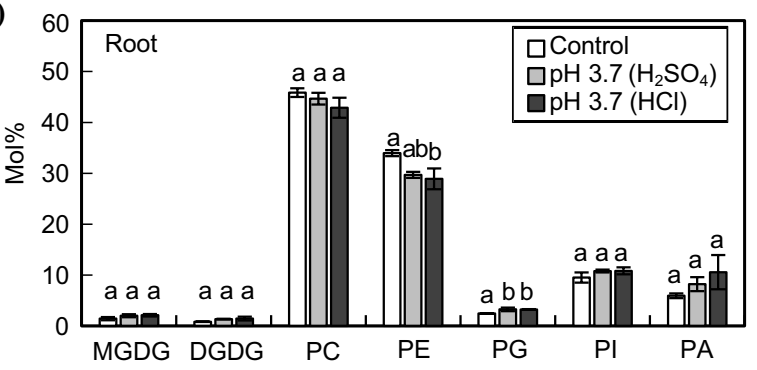

(e)

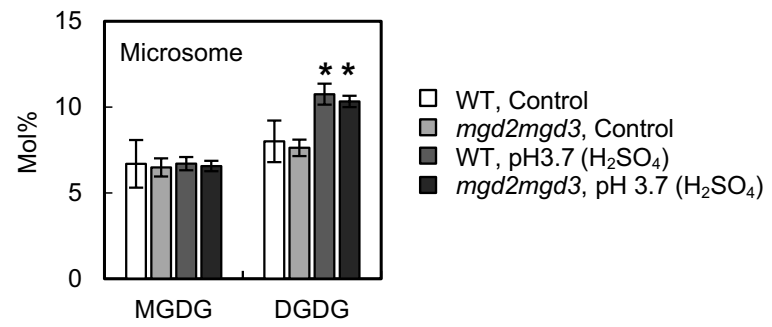

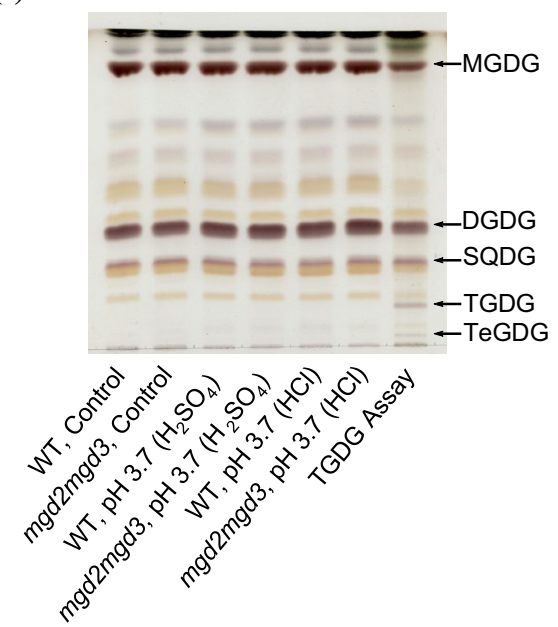

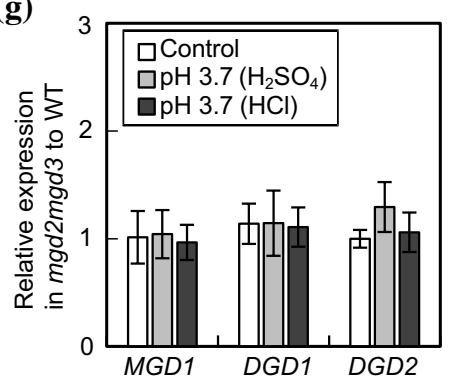

(f)

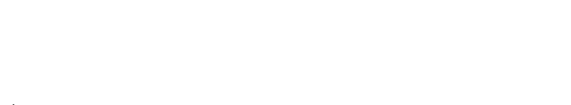

MGD1 DGD2
Fig. 3 Lipid analyses of $m g d 2 m g d 3$ plants grown under acidic conditions. Seedlings were germinated and grown as in Fig. 1. Comparison of growth phenotypes between WT and $m g d 2 m g d 3$ after $9 \mathrm{~d}$ of acidic stress treatments at the control (a) or pH 3.7 with $\mathrm{H}_{2} \mathrm{SO}_{4}(\mathbf{b})$. Bars $=1 \mathrm{~cm}$. Composition of polar membrane lipids of $m g d 2 m g d 3$ in shoots (c) and roots (d). Values are the mean \pm SD $(n=3)$. Different letters represent significant differences based on the Tukey-Kramer multiple comparison test $(p<0.05)$. e Galactolipid content in shoot microsomal membrane fractions of WT and $m g d 2 m g d 3$ plants. Values are the mean $\pm \mathrm{SD}(n=3)$. Asterisks represent significant differences from the control growth conditions ( $p<0.05$, Student's $t$ test).

$P A H 1$, represents the transcriptional level already induced by Pi starvation as compared with Pi-sufficient conditions. In rosette leaves, $M G D 3$ and At4 were upregulated under Pi-deficient conditions at $\mathrm{pH} 3.7$ as compared with $\mathrm{pH}$ 6.0, f Glycolipid staining on a thin-layer chromatogram. Sugars in head groups of lipids are visualized by the anthrone-sulfuric acid assay. As the positive control for oligogalactolipid, a 14-day-old WT Arabidopsis rosette was floated on the assay buffer $(20 \mathrm{mM}$ acetic acid, $10 \mathrm{mM}$ $\mathrm{MgCl}_{2}, \mathrm{pH}$ adjusted to 4.0 with $\mathrm{K}_{2} \mathrm{HPO}_{4}$ ) overnight (Barnes et al. 2016). g Relative expression of $M G D 1, D G D 1$ and $D G D 2$ in rosette leaves of $m g d 2 m g d 3$ to WT. Relative mRNA abundance of each gene in expanded rosette leaves was analyzed by qPCR using $U B Q 10$ as an internal control. Values are the mean $\pm \mathrm{SD}(n=6)$ of expression fold differences relative to that of the same conditions in WT

although the other genes were not significantly changed (Fig. 5c). Meanwhile, in roots, $M G D 2 / 3, D G D 1 / 2, N P C 4 / 5$ and $P A H I$ were downregulated under acidic Pi-deficient conditions, indicating that lipid remodeling during Pi 
(a)

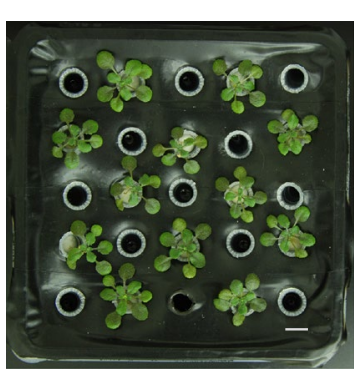

(c)

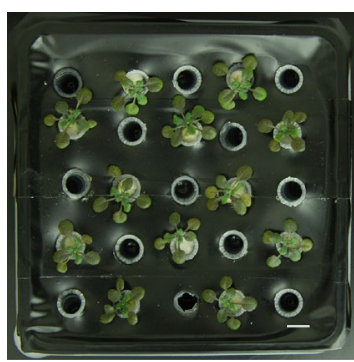

(b)

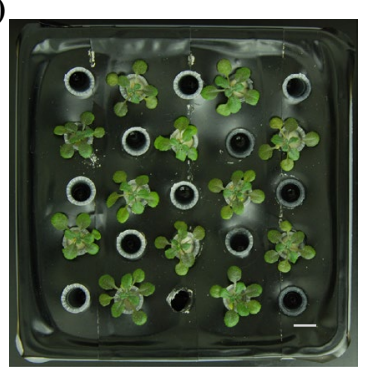

(d)

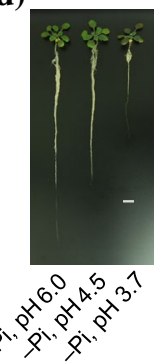

(e)

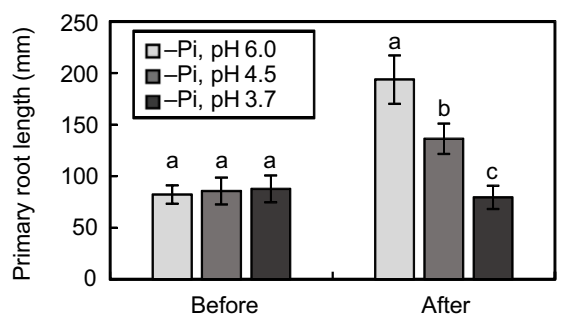

Fig. 4 Growth phenotypes of WT Arabidopsis under acidic Pi-deficient conditions. Plant seedlings were germinated on 1/2 MS medium for 10 days and transferred to 1/3 MGRL medium without phosphate (-Pi) containing $10 \mathrm{mM}$ MES-KOH (pH 6.0) for 5 days. Then, acidic Pi depletion stress treatments were subsequently conducted. Plants were grown in 1/3 MGRL medium - $\mathrm{Pi}$ at $\mathrm{pH} 6.0$ (a), 4.5 (b) or 3.7 (c) adjusted with MES-KOH (pH 6.0) or $\mathrm{H}_{2} \mathrm{SO}_{4}(\mathrm{pH} 4.5$ and 3.7) for another 7 days. d Whole morphology of WT Arabidopsis grown under acidic Pi-deficient conditions. Bars $=1 \mathrm{~cm}$. e Primary root length of WT Arabidopsis before (-Pi, 5 days) and after (-Pi, 12 days) a week of acidic Pi depletion stress treatments. Values are the mean \pm SD $(n=20)$. Different letters represent significant differences based on the Tukey-Kramer multiple comparison test $(p<0.05)$

depletion in roots is transcriptionally repressed by acidic stress (Fig. 5d). At4 and IAAl were not downregulated in roots under acidic Pi-deficient conditions. Thus, although the effect of acidic stress to the expression of Pi starvationinduced genes might be very limited, it was clearly shown that acidic stress represses expression of genes involved in $\mathrm{Pi}$ starvation-induced lipid remodeling in roots and thus represses the lipid remodeling.

Membrane lipid remodeling in roots under acidic $\mathrm{Pi}$ deficiency

Pi starvation-induced lipid remodeling in roots was partially repressed by acidic stress, but still DGDG content in Pi-starved roots at lower $\mathrm{pH}$ was higher than that of Pi-sufficient conditions (Figs. 3d, 5b). Previous research has suggested that membranes comprising glycolipids are resistant to proton permeation relative to phospholipids (Fuks and Homblé 1996; Paula et al. 1996). The proton-resisting characteristics of glycolipids are thought to result from the abundance of hydroxyl groups in the sugar moieties (Róg et al. 2007; Shimada et al. 2008; Wang et al. 2012). To compare the proton permeability of membranes comprising DGDG and PC from plants, we prepared liposomes including DGDG and/or PC extracted from spinach at three ratios (Fig. 6a). Proton permeability of these membranes was evaluated by observation of changes in the internal $\mathrm{pH}$ of liposomes after dilution of the liposome suspension (pH 7.0) with acidic buffer (pH 4.5) (Fig. 6b). Notably, the proton influx for the liposome membrane made of lecithin, PC and DGDG ( $30 \%$ DGDG) was lower than that made of lecithin and PC (0\% DGDG), and was higher than that made of lecithin and DGDG ( $\sim 60 \%$ DGDG). These results clearly indicated that a membrane comprising a plant DGDG has a lower proton permeability than that of PC in vitro.

To investigate the physiological effects of DGDG accumulation in root membranes during acidic Pi starvation stress, we compared the growth of WT Arabidopsis with that of $m g d 2 m g d 3$ because DGDG scarcely increases in roots of $m g d 2 m g d 3$ during Pi depletion (Kobayashi et al. 2009a, b). Although there were no obvious phenotypic differences between WT and $m g d 2 m g d 3$ at pH 6.0 (Fig. 7a), $m g d 2 m g d 3$ showed notably discolored rosette leaves at $\mathrm{pH} 3.7$, whereas WT did not (Fig. 7b). We also quantified the chlorophyll content in rosettes from WT and $m g d 2 m g d 3$. At $\mathrm{pH} 6.0$, the chlorophyll content in both WT and $m g d 2 m g d 3$ was similarly decreased during the time course (Fig. 7c). In contrast, at $\mathrm{pH}$ 3.7, $m g d 2 m g d 3$ showed a more rapid decrease in chlorophyll content than WT (Fig. 7d), suggesting that $m g d 2 m g d 3$ is more sensitive to acidic Pi deficiency than WT. We also analyzed the lipid composition of $m g d 2 m g d 3$ plants grown under acidic Pi-deficient conditions (Fig. 7e, f). In shoots and roots of $m g d$ $2 m g d 3$, the effect of acidic stress under Pi deficiency was not remarkable compared with the case of WT (Fig. 5a, b) and the lipid compositions of seedlings under two different $\mathrm{pH}$ conditions (-Pi, pH 6.0 and $\mathrm{pH} 3.7$ ) were not remarkably different (Fig. 7e, f). However, when compared with WT, the amount of DGDG in roots of $m g d 2 m g d 3$ was markedly lower than that of WT (Figs. 5b, 7f).

These results indicate that the function of type-B MGDG synthases and, thus, the DGDG increase triggered by Pi starvation, especially in roots, contributes to the acquisition 
(a)

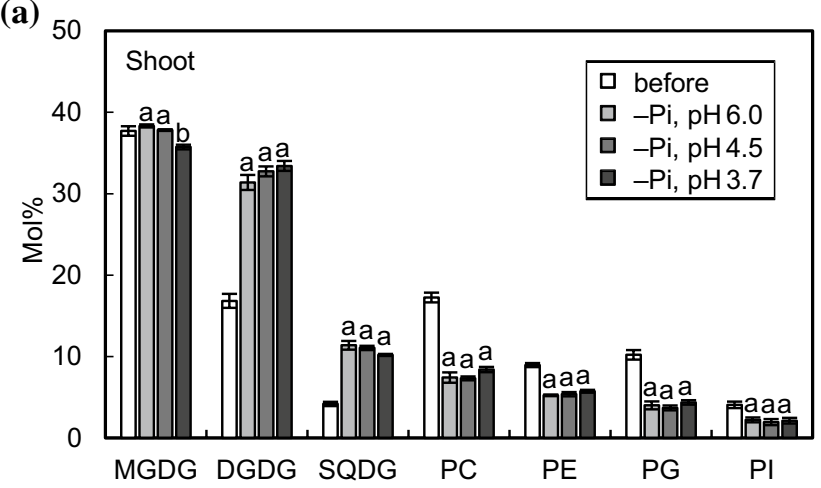

(c)
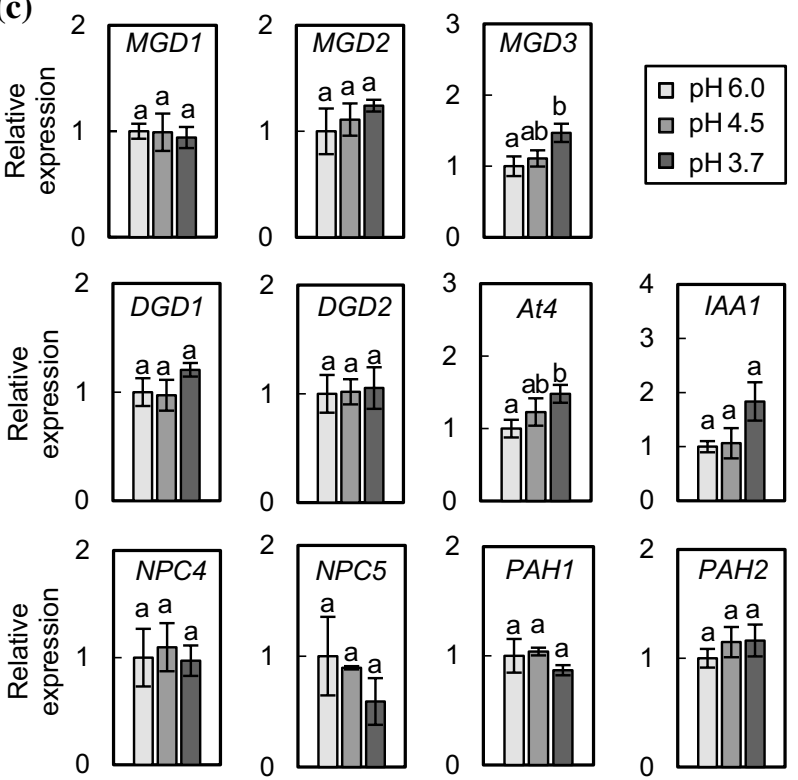

(b)

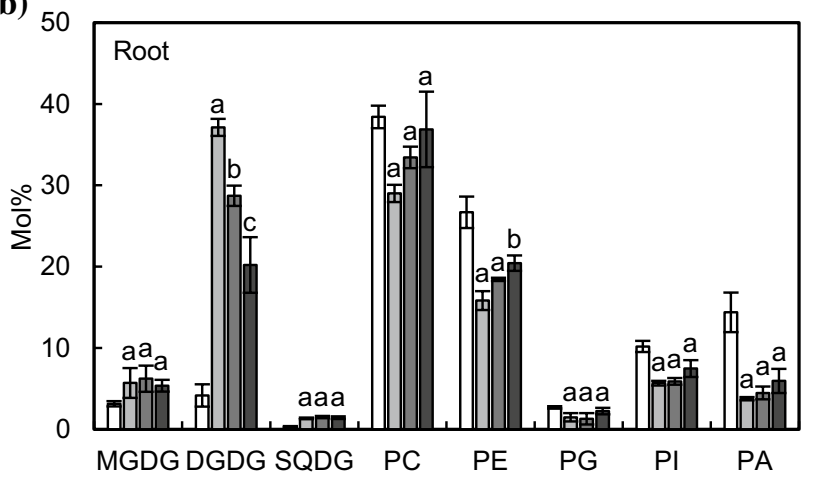

(d)
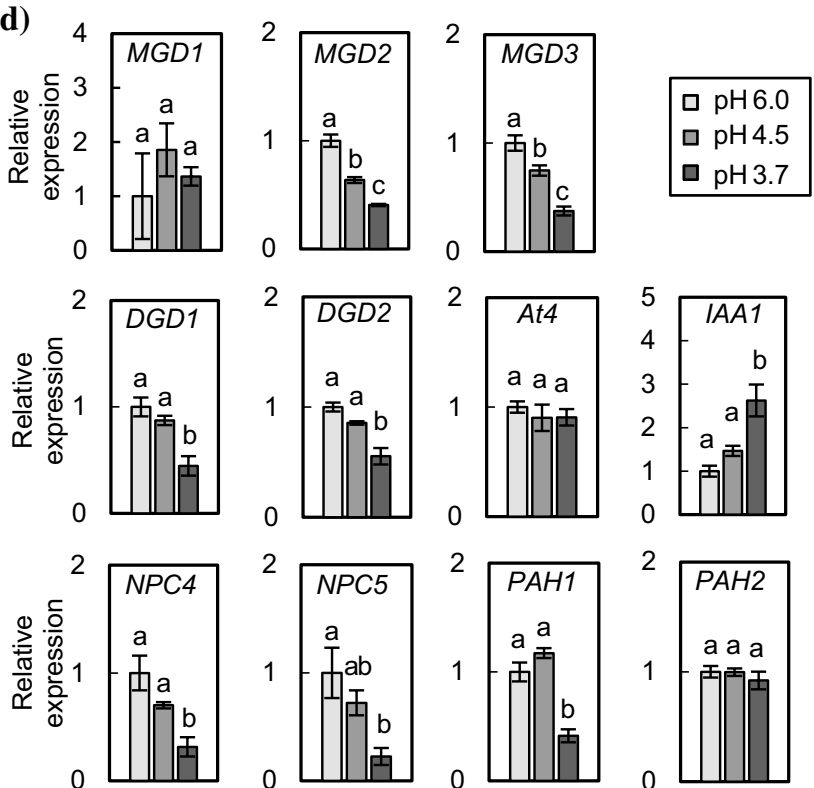

Fig. 5 Lipid and gene expression analyses of WT Arabidopsis grown under acidic Pi-deficient conditions. Seedlings were germinated and grown as in Fig. 4. Lipid composition of polar membrane lipids in shoots (a) and roots (b) was measured before (-Pi, 5 days) and after ( $-\mathrm{Pi}, 12$ days) a week of acidic Pi depletion stress treatments. Values are the mean $\pm \mathrm{SD}(n=3)$. Gene expression analyses in rosette leaves

of whole-plant stress tolerance under acidic Pi-deficient conditions.

\section{Discussion}

DGDG in shoots from both WT and $m g d 2 m g d 3$ grown at pH 3.7 showed an $\sim 25 \%$ increase in molar percentages as compared with plants grown under control conditions (Figs. 2a, 3c). From previous research, an $\sim 25 \%$ increase in DGDG can lead to stress tolerance in plants. For example, high temperature induces a DGDG increase of $23 \%$, and a partial defect in DGD1 in Arabidopsis reduces thermotolerance (Chen et al. 2006). DGDG is also increased

(c) and roots (d) of WT Arabidopsis grown under acidic Pi-deficient conditions. Relative mRNA abundance of each gene was analyzed by $\mathrm{qPCR}$ with $U B Q 10$ as an internal control. Values are the mean $\pm \mathrm{SD}$ $(n=3)$ of expression fold differences from that of $\mathrm{pH}$ 6.0. Different letters represent significant differences based on the Tukey-Kramer multiple comparison test $(p<0.05)$

by $28 \%$ in Arabidopsis during drought stress (Gigon et al. 2004). Moreover, transgenic tobacco (Nicotiana tabacum) lines that overexpress the rice MGD gene have a 11-24\% higher DGDG content as compared with WT and show enhanced salt tolerance (Wang et al. 2014). Although the mechanisms remain unclear, these results together suggest that an increase of only 25\% in DGDG possibly mediated by $D G D 1$ should be sufficient to achieve tolerance to various abiotic stresses. As the results of gene expression analyses in WT Arabidopsis grown under acidic conditions, transcriptional regulation of galactolipid metabolism during acidic stress resembled that of Pi starvation (Fig. 2c). One possibility is that auxin-cytokinin interaction is the key regulation mechanisms of galactolipid metabolism 
(a)

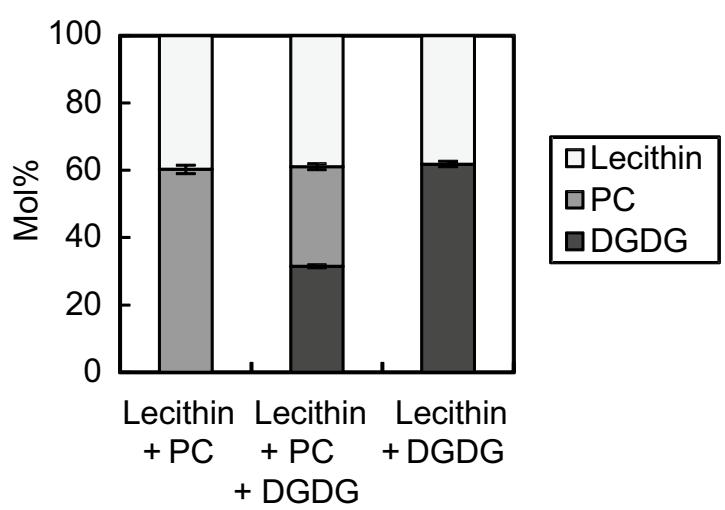

(b)

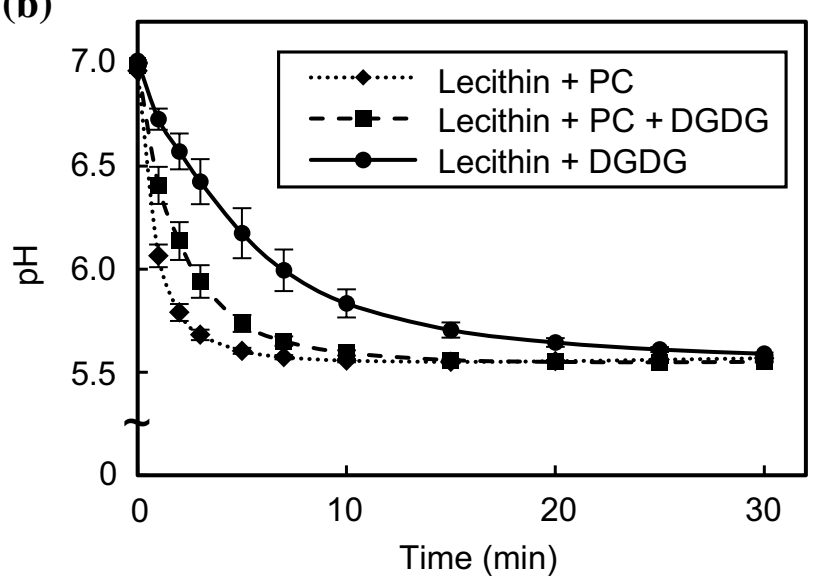

Fig. 6 Proton permeability of membranes comprising DGDG and PC from spinach. a Lipid composition of liposomes. $\mathbf{b}$ Time course of changes in internal $\mathrm{pH}$ of liposomes. The fluorescence emission at $510 \mathrm{~nm}$ of the $\mathrm{pH}$-sensitive probe pyranine present within the liposome was measured at 1, 2, 3, 5, 7, 10, 15, 20, 25 and 30 min after $100 \mu \mathrm{L}$ of liposome suspension ( $\mathrm{pH} 7.0$ ) was mixed with $1.9 \mathrm{~mL}$ of acidic buffer ( $\mathrm{pH} 4.5)$. For the intensity at $0 \mathrm{~min}$, the liposome suspension was diluted with neutral buffer $(\mathrm{pH} 7.0)$. Internal $\mathrm{pH}$ was calculated based on the calibration curve showing the relationship between solution $\mathrm{pH}$ and the logarithm of the ratio of the emission intensity with excitation at 400 to that at $460 \mathrm{~nm}$ (Figure S5). Values are the mean $\pm \mathrm{SD}(n=3)$

responding to acidic stress. Low-pH stress induces the accumulation of auxin and a reduction in cytokinins in Arabidopsis shoots (Hachiya et al. 2014). Auxin signaling is involved in activation of $M G D 2 / 3$ and lipid remodeling during Pi depletion (Kobayashi et al. 2006; Narise et al. 2010). Conversely, exogenous cytokinin treatments repress DGDG accumulation even under Pi deficiency, especially in root membrane (Kobayashi et al. 2006). In addition, $M G D 1$ is transcriptionally up-regulated via cytokinin signaling (Yamaryo et al. 2003; Shimojima and Ohta 2011). Thus, transcriptional upregulation of $M G D 2 / 3$ concomitant with downregulation of $M G D 1$ under acidic conditions might be achieved by an auxin-cytokinin interaction. Although both $M G D 2$ and $M G D 3$ were transcriptionally (a)

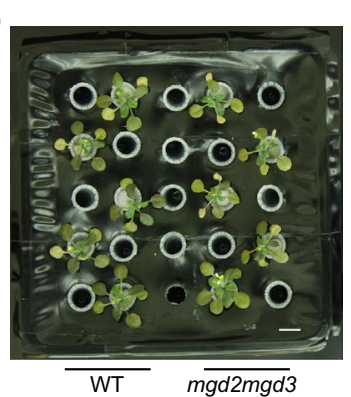

(c)

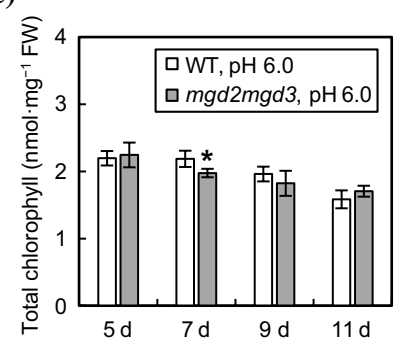

(b)

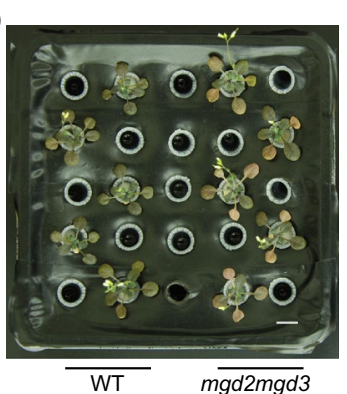

(d)

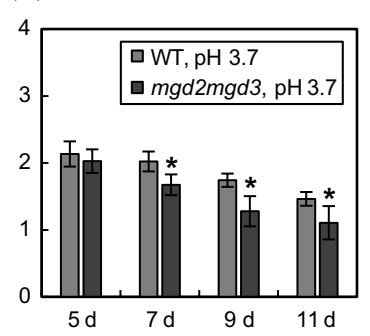

(e)

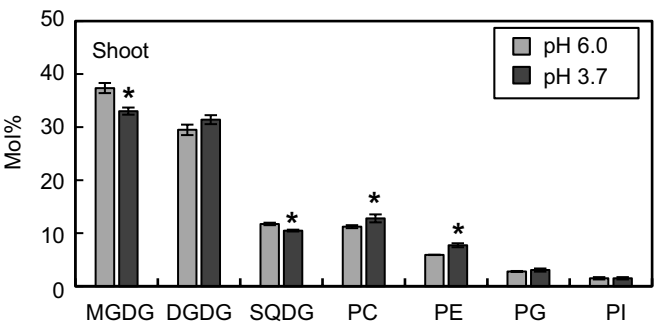

(f)

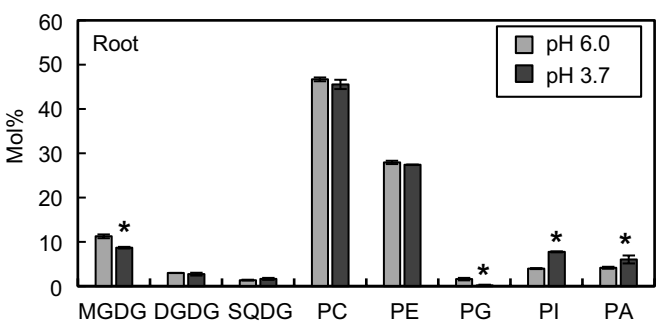

Fig. 7 Comparison of phenotypes of WT Arabidopsis and $m g d 2 m g d 3$ under acidic Pi-deficient conditions. Seedlings were germinated and grown as in Fig. 4. Comparison of growth phenotypes between WT and $m g d 2 m g d 3$ after 9 days of acidic Pi depletion stress treatments at pH 6.0 (a) or 3.7 (b). Bars $=1 \mathrm{~cm}$. Chlorophyll content in rosettes from WT and $m g d 2 m g d 3$ after 5, 7, 9 and 11 days of acidic Pi depletion stress treatments at $\mathrm{pH} 6.0$ (c) or 3.7 (d). Values are the mean $\pm \mathrm{SD}(n=4)$. Asterisks represent significant differences between WT and $m g d 2 m g d 3$ for each time point ( $p<0.05$, Student's $t$-test). Lipid composition of polar membrane lipids in shoots (e) and roots (f) of $m g d 2 m g d 3$ was measured after 7 days of acidic Pi depletion stress treatments at $\mathrm{pH} 6.0$ or 3.7. Values are the mean $\pm \mathrm{SD}(n=3)$. Asterisks represent significant differences between $\mathrm{pH} 6.0$ and $\mathrm{pH} 3.7$ for each lipid ( $p<0.05$, Student's $t$-test)

upregulated by acidic stress, $m g d 2 m g d 3$ showed a comparable increase of DGDG in shoots relative to WT under acidic conditions (Figs. 2a, 3c), indicating that MGD1DGD1 pathway is responsible for DGDG accumulation in shoots under acidic conditions. The relative expression 
of NPC4/5, which supply diacylglycerol for the MGD2/3DGD2 pathway during Pi depletion (Nakamura et al. 2005; Gaude et al. 2008), was comparable at pH 3.7 and control conditions, whereas that of $P A H 1$, which supplies the substrate for the MGD1-DGD1 pathway (Nakamura et al. 2009b), was upregulated at pH 3.7 (Fig. 2c). Lack of enough substrate supply might be the cause of non-engagement of type-B MGDs in DGDG accumulation during acidic stress. Moreover, it should also be considered for clarifying the regulatory mechanism of MGDG synthesis under acidic conditions that MGD activity is regulated in a redox-dependent manner (Yamaryo et al. 2006), and that jasmonic acid biosynthesis and signaling were involved in MGDG steady state level by regulating MGD1 activity and MGD3 expression (Chevalier et al. 2019).

Phospholipids in membrane are thought to constitute up to one-third of total Pi source in plant cell. Thus, replacing phospholipids with non-phosphorus lipids can be advantageous in growth enhancement during Pi depletion. We showed that DGDG increase in root membranes during Pi depletion is partially repressed at a lower $\mathrm{pH}$ while other $\mathrm{Pi}$ starvation responses, such as expression of At4 and the auxin signaling component $I A A I$, are maintained (Fig. 5b, d). In addition, preferential root growth during Pi depletion was inhibited at a lower $\mathrm{pH}$, in parallel with the repression of membrane lipid remodeling (Fig. 4e). The increase in surface area caused by root growth enhancement could be beneficial for taking up nutrients under Pi deficiency but also would be harmful in low-pH environments. The results suggest that plants optimize root growth by controlling the membrane lipid remodeling to adapt to the pleiotropic stress effects of acidic Pi deficiency.

Membrane lipid remodeling during Pi depletion has also been considered to play a role in $\mathrm{Al}^{3+}$ tolerance. $\mathrm{Ca}^{2+}$ usually stabilizes plasma membrane by binding to the negative charge on the membrane, mainly the phosphate group of phospholipids. $\mathrm{Al}^{3+}$, which is one of the most rhizotoxic ions in acid soils in nature, is thought to destabilize cell membranes by replacing membrane-bound $\mathrm{Ca}^{2+}$ (Kobayashi et al. 2013). Actually, pah1pah2 double mutant includes more phospholipids in cell membranes than WT Arabidopsis (Nakamura et al. 2009b) and is more sensitive to $\mathrm{Al}^{3+}$ than WT during Pi depletion (Kobayashi et al. 2013). Moreover, induction of membrane lipid remodeling by $\mathrm{Pi}$ depletion enhances $\mathrm{Al}^{3+}$ tolerance in rice (Maejima and Watanabe 2014). Similar to $\mathrm{Al}^{3+}$, proton is also thought to destabilize cell membranes (Kobayashi et al. 2013). In this study, we showed that liposome membranes that include DGDG obtained from plants have characteristics of a proton barrier in vitro (Fig. 6b). In addition, the $m g d 2 m g d 3$ double mutant, in which membrane lipid remodeling in roots is deficient, showed impairment of adaptation to acidic Pi deficiency (Fig. 7), suggesting the advantage of galactolipid-enriched extraplastidic membranes in roots under acidic conditions. A greater understanding of the physicochemical and biological characteristics of membranes comprising galactolipid and phospholipid is required to clarify the physiological significance of membrane lipid alterations in plants grown under various complicated environmental stresses. Further analyses under various combined stress conditions will provide us new insights into stress adaptation mechanisms in plants.

Acknowledgements We thank Akira Nozawa and Hiroyuki Inoue of Ehime University for providing lecithin and giving technical advice on making liposomes.

Author Contribution HO and MS supervised the experiments; MM performed most of the experiments; MM and MS designed the experiments and analyzed the data; MM conceived the project and wrote the article with contributions from all the authors; HO and MS supervised the writing.

Funding A Grants-in-Aid for Scientific Research on Innovative Areas (Nos. 255119708 and 17H06417); A Grant-in-Aid for Scientific Research (C) (No. 15K07335) from the Ministry of Education, Culture, Sports, Science and Technology of Japan; Core Research for Evolutional Science and Technology programs of the Japanese Science and Technology Agency.

Open Access This article is distributed under the terms of the Creative Commons Attribution 4.0 International License (http://creativeco mmons.org/licenses/by/4.0/), which permits unrestricted use, distribution, and reproduction in any medium, provided you give appropriate credit to the original author(s) and the source, provide a link to the Creative Commons license, and indicate if changes were made.

\section{References}

Andersson MX, Stridh MH, Larsson KE, Liljenberg C, Sandelius AS (2003) Phosphate-deficient oat replaces a major portion of the plasma membrane phospholipids with the galactolipid digalactosyldiacylglycerol. FEBS Lett 537:128-132

Andersson MX, Larsson KE, Tjellström H, Liljenberg C, Sandelius AS (2005) Phosphate-limited oat: the plasma membrane and the tonoplast as major targets for phospholipid-to-glycolipid replacement and stimulation of phospholipases in the plasma membrane. J Biol Chem 280:27578-27586

Awai K, Maréchal E, Block MA, Brun D, Masuda T, Shimada H, Takamiya K, Ohta H, Joyard J (2001) Two types of MGDG synthase genes, found widely in both 16:3 and 18:3 plants, differentially mediate galactolipid syntheses in photosynthetic and nonphotosynthetic tissues in Arabidopsis thaliana. Proc Natl Acad Sci USA 98:10960-10965

Bari R, Pant BD, Stitt M, Scheible WR (2006) $\mathrm{PHO}_{2}$, microRNA399, and PHR1 define a phosphate-signaling pathway in plants. Plant Physiol 141:988-999

Barnes AC, Benning C, Roston R (2016) Chloroplast membrane remodeling during freezing stress is accompanied by cytoplasmic acidification activating SENSITIVE TO FREEZING2. Plant Physiol 171:2140-2149

Benning C, Ohta H (2005) Three enzyme systems for galactoglycerolipid biosynthesis are coordinately regulated in plants. J Biol Chem 280:2397-2400 
Bligh EG, Dyer WJ (1959) A rapid method of total lipid extraction and purification. Can J Biochem Physiol 37:911-917

Block MA, Dorne A-J, Joyard J, Douce R (1983) Preparation and characterization of membrane fractions enriched in outer and inner envelope membranes from spinach chloroplasts. J Biol Chem 258:13281-13286

Chen J, Burke JJ, Xin Z, Xu C, Velten J (2006) Characterization of the Arabidopsis thermosensitive mutant atts02 reveals an important role for galactolipids in thermotolerance. Plant Cell Environ 29:1437-1448

Chevalier F, Cuyas L, Jouhet J, Gros V, Chiarenza S, Secco D, Whelan J, Seddiki K, Block MA, Nussaume L, Maréchal E (2019) Interplay between jasmonic acid, phosphate signaling and the regulation of glycerolipid homeostasis in Arabidopsis. Plant Cell Physiol 60(6):1260-1273

Chiou TJ, Aung K, Lin SI, Wu CC, Chiang SF, Su CL (2006) Regulation of phosphate homeostasis by MicroRNA in Arabidopsis. Plant Cell 18:412-421

Dörmann P, Benning C (2002) Galactolipids rule in seed plants. Trends Plant Sci 7:112-118

Fujiwara T, Hirai MY, Chino M, Komeda Y, Naito S (1992) Effects of sulfur nutrition on expression of the soybean seed storage protein genes in transgenic petunia. Plant Physiol 99:263-268

Fuks B, Homblé F (1996) Mechanism of proton permeation through chloroplast lipid membranes. Plant Physiol 112:759-766

Gaude N, Nakamura Y, Scheible WR, Ohta H, Dörmann P (2008) Phospholipase C5 (NPC5) is involved in galactolipid accumulation during phosphate limitation in leaves of Arabidopsis. Plant J 56:28-39

Gigon A, Matos AR, Laffray D, Zuily-Fodil Y, Pham-Thi AT (2004) Effect of drought stress on lipid metabolism in the leaves of Arabidopsis thaliana (Ecotype Columbia). Ann Bot 94:345-351

Hachiya T, Sugiura D, Kojima M, Sato S, Yanagisawa S, Sakakibara $\mathrm{H}$, Terashima I, Noguchi K (2014) High $\mathrm{CO}_{2}$ triggers preferential root growth of Arabidopsis thaliana via two distinct systems under low $\mathrm{pH}$ and low N stresses. Plant Cell Physiol 55:269-280

Härtel H, Dormann P, Benning C (2000) DGD1-independent biosynthesis of extraplastidic galactolipids after phosphate deprivation in Arabidopsis. Proc Natl Acad Sci USA 97:10649-10654

Hinsinger P, Plassard C, Tang C, Jaillard B (2003) Origins of rootmediated $\mathrm{pH}$ changes in the rhizosphere and their responses to environmental constraints: a review. Plant Soil 248:43-59

Inoue SI, Takahashi K, Okumura-Noda H, Kinoshita T (2016) Auxin influx carrier AUX1 confers acid resistance for Arabidopsis root elongation through the regulation of plasma membrane $\mathrm{H}^{+}$-ATPase. Plant Cell Physiol 57:2194-2201

Jouhet J, Maréchal E, Baldan B, Bligny R, Joyard J, Block MA (2004) Phosphate deprivation induces transfer of DGDG galactolipid from chloroplast to mitochondria. J Cell Biol 167:863-874

Kano K, Fendler JH (1978) Pyranine as a sensitive $\mathrm{pH}$ probe for liposome interiors and surfaces. $\mathrm{pH}$ gradients across phospholipid vesicles. Biochim Biophys Acta 509:289-299

Kelly AA, Dörmann P (2002) DGD2, an Arabidopsis gene encoding a UDP-galactose-dependent digalactosyldiacylglycerol synthase is expressed during growth under phosphate-limiting conditions. J Biol Chem 277:1166-1173

Kelly AA, Froehlich JE, Dörmann P (2003) Disruption of the two digalactosyldiacylglycerol synthase genes DGD1 and DGD2 in Arabidopsis reveals the existence of an additional enzyme of galactolipid synthesis. Plant Cell 15:2694-2706

Kobayashi K, Masuda T, Takamiya K, Ohta H (2006) Membrane lipid alteration during phosphate starvation is regulated by phosphate signaling and auxin/cytokinin cross-talk. Plant J 47:238-248

Kobayashi K, Kondo M, Fukuda H, Nishimura M, Ohta H (2007) Galactolipid synthesis in chloroplast inner envelope is essential for proper thylakoid biogenesis, photosynthesis, and embryogenesis. Proc Natl Acad Sci USA 104:17216-17221

Kobayashi K, Awai K, Nakamura M, Nagatani A, Masuda T, Ohta H (2009a) Type-B monogalactosyldiacylglycerol synthases are involved in phosphate starvation-induced lipid remodeling, and are crucial for low-phosphate adaptation. Plant J 57:322-331

Kobayashi K, Nakamura Y, Ohta H (2009b) Type A and type B monogalactosyldiacylglycerol synthases are spatially and functionally separated in the plastids of higher plants. Plant Physiol Biochem 47:518-525

Kobayashi Y, Kobayashi Y, Watanabe T, Shaff JE, Ohta H, Kochian LV, Wagatsuma T, Kinraide TB, Koyama H (2013) Molecular and physiological analysis of $\mathrm{Al}^{3+}$ and $\mathrm{H}^{+}$rhizotoxicities at moderately acidic conditions. Plant Physiol 163:180-192

Koyama H, Toda T, Hara T (2001) Brief exposure to low-pH stress causes irreversible damage to the growing root in Arabidopsis thaliana: pectin-Ca interaction may play an important role in proton rhizotoxicity. J Exp Bot 52:361-368

Lager I, Andréasson O, Dunbar TL, Andreasson E, Escobar MA, Rasmusson AG (2010) Changes in external $\mathrm{pH}$ rapidly alter plant gene expression and modulate auxin and elicitor responses. Plant Cell Environ 33:1513-1528

Maejima E, Watanabe T (2014) Proportion of phospholipids in the plasma membrane is an important factor in Al tolerance. Plant Signal Behav 9:e29277

Martín AC, Del Pozo JC, Iglesias J, Rubio V, Solano R, De La Peña A, Leyva A, Paz-Ares J (2000) Influence of cytokinins on the expression of phosphate starvation responsive genes in Arabidopsis. Plant J 24:559-567

Miège C, Maréchal E, Shimojima M, Awai K, Block MA, Ohta H, Takamiya K, Douce R, Joyard J (1999) Biochemical and topological properties of type A MGDG synthase, a spinach chloroplast envelope enzyme catalyzing the synthesis of both prokaryotic and eukaryotic MGDG. Eur J Biochem 265:990-1001

Moellering ER, Muthan B, Benning C (2010) Freezing tolerance in plants requires lipid remodeling at the outer chloroplast membrane. Science 330:226-228

Murakawa M, Shimojima M, Shimomura Y, Kobayashi K, Awai K, Ohta H (2014) Monogalactosyldiacylglycerol synthesis in the outer envelope membrane of chloroplasts is required for enhanced growth under sucrose supplementation. Front Plant Sci 5:280

Murashige T, Skoog F (1962) A revised medium for rapid growth and bio assays with tobacco tissue cultures. Plant Physiol 15:473-497

Nakamura Y (2013) Phosphate starvation and membrane lipid remodeling in seed plants. Prog Lipid Res 52:43-50

Nakamura Y, Awai K, Masuda T, Yoshioka Y, Takamiya K, Ohta H (2005) A novel phosphatidylcholine-hydrolyzing phospholipase $\mathrm{C}$ induced by phosphate starvation in Arabidopsis. J Biol Chem 280:7469-7476

Nakamura Y, Kobayashi K, Ohta H (2009a) Activation of galactolipid biosynthesis in development of pistils and pollen tubes. Plant Physiol Biochem 47:535-539

Nakamura Y, Koizumi R, Shui G, Shimojima M, Wenk MR, Ito T, Ohta $\mathrm{H}$ (2009b) Arabidopsis lipins mediate eukaryotic pathway of lipid metabolism and cope critically with phosphate starvation. Proc Natl Acad Sci USA 106:20978-20983

Narise T, Kobayashi K, Baba S, Shimojima M, Masuda S, Fukaki H, Ohta H (2010) Involvement of auxin signaling mediated by IAA14 and ARF7/19 in membrane lipid remodeling during phosphate starvation. Plant Mol Biol 72:533-544

Paula S, Volkov AG, Van Hoek AN, Haines TH, Deamer DW (1996) Permeation of protons, potassium ions, and small polar molecules through phospholipid bilayers as a function of membrane thickness. Biophys J 70:339-348 
Porra RJ (2002) The chequered history of the development and use of simultaneous equations for the accurate determination of chlorophylls a and b. Photosynth Res 73:149-156

Róg T, Vattulainen I, Bunker A, Karttunen M (2007) Glycolipid membranes through atomistic simulations: effect of glucose and galactose head groups on lipid bilayer properties. J Phys Chem B 111:10146-10154

Shaff JE, Schultz BA, Craft EJ, Clark RT, Kochian LV (2010) GEOCHEM-EZ: a chemical speciation program with greater power and flexibility. Plant Soil 330:207-214

Shavrukov Y, Hirai Y (2016) Good and bad protons: genetic aspects of acidity stress responses in plants. J Exp Bot 67:15-30

Shimada H, Nemoto N, Shida Y, Oshima T, Yamagishi A (2008) Effects of $\mathrm{pH}$ and temperature on the composition of polar lipids in Thermoplasma acidophilum HO-62. J Bacteriol 190:5404-5411

Shimojima M, Ohta H (2011) Critical regulation of galactolipid synthesis controls membrane differentiation and remodeling in distinct plant organs and following environmental changes. Prog Lipid Res 50:258-266

von Uexküll HR, Mutert E (1995) Global extent, development and economic impact of acid soils. Plant Soil 171:1-15

Wang X, Lv B, Cai G, Fu L, Wu Y, Wang X, Ren B, Ma H (2012) A proton shelter inspired by the sugar coating of acidophilic archaea. Sci Rep 2:892

Wang S, Uddin MI, Tanaka K, Yin L, Shi Z, Qi Y, Mano J, Matsui K, Shimomura N, Sakaki T, Deng X, Zhang S (2014) Maintenance of chloroplast structure and function by overexpression of the rice monogalactosyldiacylglycerol synthase gene leads to enhanced salt tolerance in tobacco. Plant Physiol 165:1144-1155

Yamaryo Y, Kanai D, Awai K, Shimojima M, Masuda T, Shimada H, Takamiya K, Ohta H (2003) Light and cytokinin play a co-operative role in MGDG synthesis in greening cucumber cotyledons. Plant Cell Physiol 44:844-855

Yamaryo Y, Motohashi K, Takamiya K-I, Hisabori T, Ohta H (2006) In vitro reconstitution of monogalactosyldiacylglycerol (MGDG) synthase regulation by thioredoxin. FEBS Lett 580:4086-4090

Yan F, Feuerle R, Schäffer S, Fortmeier H, Schubert S (1998) Adaptation of active proton pumping and plasmalemma ATPase activity of corn roots to low root medium pH. Plant Physiol 117:311-319

Zhang YK, Zhu DF, Zhang YP, Chen HZ, Xiang J, Lin XQ (2015) Low $\mathrm{pH}$-induced changes of antioxidant enzyme and ATPase activities in the roots of rice (Oryza sativa L.) seedlings. PLoS ONE 10(2):e0116971

Publisher's Note Springer Nature remains neutral with regard to jurisdictional claims in published maps and institutional affiliations. 